\title{
LES ANTIOXYGËNES ET LA CONSERVATION DES PRODUITS LAITIERS
}

par

\author{
J. ADDA
}

Station centrale de recherches laitières

et de technologie des produits animaux, Jouy en Josas (S. \& O.)

\section{I. - INTRODUGTION}

La qualité des produits laitiers, et par suite la facilité avec laquelle ils sont commercialisés et consommés, est fortement influencée par l'extrême sensibilité de la fraction lipidique à l'action de l'oxygène. Cette fragilité, génératrice de défauts de goût, est aggravée par la nécessité de "reporter » la consommation des produits dans le temps et dans l'espace.

Cette obligation a conduit à préconiser, - et à utiliser avec des fortunes diverses - , la conservation par le froid, l'emballage hermétique, le conditionnement sous atmosphère inerte.

A la suite des travaux de Moureu et Durraisse (1922), il est apparu que l'on pouvait espérer atténuer la dégradation, qu'est l'oxydation, par l'addition de composés naturels ou synthétiques, à propriétés antioxydantes.

Depuis lors, leur emploi s'est répandu, aux Etats-Unis surtout, pour la protection des matières grasses alimentaires ou pour les aliments qui contiennent une certaine proportion de matières grasses. De nombreux auteurs ont étudié les corps gras industriels ("shortenings », margarine). Par contre les lipides du lait n'ont suscité que relativement peu de travaux et c'est à ces derniers, concernant les possibilités d'emploi des antioxygènes dans le lait et ses dérivés, que nous limiterons cet exposé. Après avoir rappelé les propriétés et la nature des antioxygènes, nous examinerons successivement l'utilisation de chacun d'eux dans le lait, le lait en poudre, la crème et le beurre.

\section{II. - MODE D'ACTION DES ANTIOXYGÈNES}

\section{1 - Phénomène d'autoxydation.}

Les acides gras insaturés de la matière grasse s'autoxydent suivant une réaction en chaîne où interviennent des radicaux libres. Le processus est déclenché par l'arrachement d'un atome d'hydrogène à un groupe $\alpha$ méthyle. Le radical ainsi formé, s'unissant à une molécule d'oxygène, se transforme en radical hydrope- 
roxyde qui capte un atome d'hydrogène, appartenant à un autre groupe méthyle, pour donner une molécule d'hydroperoxyde et un nouveau radical activé (SPETSIG, 1960).

$$
-\mathrm{CH}-\mathrm{CH}=\mathrm{CH}-+\mathrm{O}_{2} \rightarrow-\underset{\text { OO. }}{\mathrm{CH}}-\mathrm{CH}=\mathrm{CH}-
$$

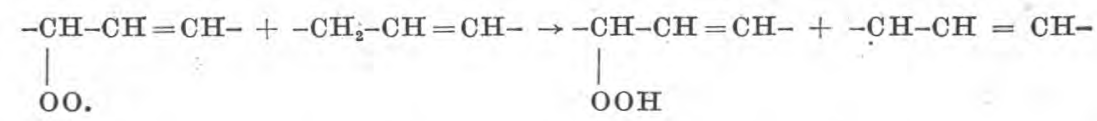

La décomposition ultérieure des molécules d'hydroperoxyde donne naissance à des aldéhydes et à des cétones ( $\left.\mathrm{DAY}_{\mathrm{AY}}, 1960\right)$ qui sont à l'origine des saveurs désagréables qui apparaissent pendant la période de conservation en chambre frigorifique.

Un antioxygène incorporé à la graisse brisera la réaction en chaîne en fournissant un atome d'hydrogène, ce qui évite la mise en jeu d'un autre groupement $\alpha$ méthyle.

Un antioxygène est donc un corps ayant une configuration électronique telle qu'elle lui permette de jouer le rôle de donneur d'hydrogène, c'est-à-dire de s'oxyder en lieu et place de la matière grasse. Une telle structure se rencontre en particulier chez les polyphénols, auxquels se rattachent la plupart des antioxydants natu. rels ou synthétiques.

$\mathrm{Au}$ contraire, les sels de métaux lourds (cuivre, fer) agissent comme pro-oxygènes. Ils facilitent l'apparition de radicaux libres à partir des hydroperoxydes, selon le schéma de la réaction indiquée ci-dessus.

\section{Différents types d'antioxygènes}

LEA (1949) distingue trois types d'antioxygènes :

- les antioxygènes primaires : ce sont en général des polyphénols substitués ou des arylamines ;

- les synergétiques et les inhibiteurs de métaux : acides ascorbique, citrique, phosphorique ;

- les antioxygènes à plusieurs fonctions.

MatTill (1945) reprenant ses travaux antérieurs (OLcotT et Mattill, 1936), ne distingue que deux types:

- les antioxygènes,

- les synergétiques. 


\section{Synergie}

Il a été prouvé que si deux antioxygènes primaires sont utilisés conjointement, le pouvoir antioxydant résultant peut être supérieur à la somme des pouvoirs antioxydants des composés utilisés séparément. On dit qu'il y a une action synergique. Toutefois un synergétique vrai est un composé qui, utilisé seul, n'a aucune action antioxydante, mais qui au contact d'un antioxygène primaire, en exalte les propriétés. Le mécanisme de cette exaltation doit résider, penset-on, dans le transfert d'atomes d'hydrogène entre les deux composés Ce phénomène d'oxydo-réduction a pour résultat de régénérer l'antioxygène primaire. Ainsi GoLumbic (1946) explique l'effet de synergie entre les polyphénols et l'acide ascorbique. Le polyphénol, oxydé en quinone, est régénéré sous sa forme phénolique grâce au rôle réducteur du synergétique qui ne fait jouer un rôle antioxydant que vis-à-vis de l'antioxygène.

\section{TABLEAU I}

LISTE DES ANTIOXYGENES AUTORISÉS PAR DIFFÉRENTES LEGISLATIONS (DOSE MAXIMUM AUTORISÉE EN P. 100)

\begin{tabular}{|c|c|c|c|c|c|}
\hline U.S.A. & & Royaume-Uni & & France (1) & \\
\hline $\begin{array}{l}\text { Gomme gaïac } \ldots \ldots \\
\text { Tocophérols } \ldots \ldots \ldots \\
\text { N D G A } \ldots \ldots \ldots \ldots \\
\text { B H A } \ldots \ldots \ldots \ldots \\
\text { B H T } \ldots \ldots \ldots \ldots \ldots \\
\text { Gallate de propyle } \ldots \\
\text { T H B P } \ldots \ldots \ldots \ldots \\
\text { Synergétiques } \ldots \ldots \ldots\end{array}$ & $\begin{array}{l}0,1 \\
0,03 \\
0,01 \\
0,01 \\
0,01 \\
0,01 \\
0,01 \\
0,01\end{array}$ & $\begin{array}{l}\text { Gallate de propyle .. } \\
\text { Gallate d'octyle } \ldots . \\
\text { Gallate de dodecyle } \\
\text { B H A . . . . . . . . . . } \\
\text { B H T } \ldots\end{array}$ & $\begin{array}{l}0,01 \\
0,01 \\
0,01 \\
0,02 \\
0,02\end{array}$ & $\begin{array}{l}\text { Gallate de propyle ... } \\
\text { Gallate d'octyle } \ldots . . \\
\text { Gallate de dodecyle . } \\
\text { B H T ........... }\end{array}$ & $\begin{array}{l}0,01 \\
0,01 \\
0,01 \\
0,01\end{array}$ \\
\hline
\end{tabular}

(1) Arrêté du 21 mai $1953(J . O$. du 10 juin 1953) autorisant l'acide ascorbique à la dose maximum de 0,03 p. 100 .

Arrêté du 3 septembre 1959 ( $J$. O. du 20 septembre 1959) concernant les gallates et le B.H.T.

Ce phénomène est mis à profit dans divers produits commerciaux qui sont constitués par des mélanges d'antioxygènes et de synergétiques.

\section{Principaux antioxygènes}

Dans la suite de cet exposé, nous classerons les anti-oxygènes d'après leur origine (naturelle ou synthétique) et nous examinerons des produits, dont l'addition n'est pas autorisée par la législation 
française, mais dont on fait usage aux Etats-Unis ou que proposent certains auteurs.

A titre indicatif, nous donnons dans le tableau I la liste des produits dont l'adjonction est autorisée par les législation américaine, britannique et française.

\section{III. - UTILISATION DES ANTIOXYGÈNES}

\section{1 - Antioxygènes naturels.}

\section{Acide Nordihydroguaiarétique (NDGA)}

Le 4-4' (2-3) diméthyltétraméthylène dipyrocatéchol connu sous le nom d'acide nordihydroguaiarétique a été proposé comme comme antioxygène par LUNDBERG, HaLvorson et BURR (1944). Ce composé est extrait d'une plante de la famille des Zygophyllées : Larrea divaricata.

Stull, Herreid et Tracy (1948a) constatent qu'un lait entier, contenant de 0,00125 à 0,0075 p. 100 de NDGA, peut être conservé einq jours à la température de $+4^{\circ} \mathrm{C}$ sans apparition de saveur oxydée. Trout et Weinstein (1953) exposent un lait homogénéisé, additionné de 0,0025 p. 100 de NDGA, pendant $60 \mathrm{mn}$ à la lumière solaire, sans qu'une saveur oxydée en résulte ; mais s'ils augmentent la concentration en antioxygène jusqu'à $0,0075 \mathrm{p}$. 100 le lait prend un goût amer. L'addition préalable de 0,0035 p. 100 d'acide ascorbique empêche l'apparition de cette amertume.

L'addition de faibles quantités de NDGA $(0,1$ p. 100 calculé sur la poudre) à des laits destinés à être transformés en poudre de lait, permet d'améliorer les qualités de conservation de la poudre ; ainsi Stull, Herreid et Tracy (1951) conservent une poudre Spray pendant 12 mois à la température du laboratoire, sans apparition de saveur oxydée; mais TollenaAR (1952) signale qu'un tel lait reconstitué et porté à l'ébullition prend un goût amer.

La solubilité du NDGA dans les corps gras permet de l'incorporer facilement aux crèmes et aux beurres.

Selon KRUKovsky et al. (1949), l'antioxygène incorporé au lait avant pasteurisation, à raison de $0,005 \mathrm{p}$. 100 a permis de conserver de façon satisfaisante une crème soumise successivement à un séjour de 30 jours à la température de $0^{\circ} \mathrm{C}$ puis à un séjour de 15 à 247 jours à la température de $-16^{\circ} \mathrm{C}$.

Stull, Herreid et Tracy (1948b) montrent qu'à la même concentration le NDGA permet de conserver des crèmes acides et des crèmes douces pendant des laps de temps atteignant 11 et 12 mois. 
Par contre, l'addition du NDGA au beurre s'est révélée peu avantageuse. En l'ajoutant à raison de 0,001 p. 100 SwartLing (1949) mesure des indices de peroxyde inférieurs à ceux des témoins, bien qu'il y ait apparition d'un goût désagréable qualifié de " graisseux " (oily flavour). A la dose où il est efficace $(0,005$ p. 100) l'antioxygène se trahit par sa saveur propre (SwartLING, 1949 ; New Zealand Dairy Res. Inst., 1949 ; Mac Dowell, 1955).

D'après d'autres expériences (ULEX et KRöGER, 1950; VAN Haeften et Pette 1953) ce composé ne produirait que peu ou pas d'effets favorables.

\section{Quercétine}

La quercétine qui est un flavonol, semble présenter une activité antioxydante. TARASSUK et HENDERSON (1946) estiment qu'à très faible concentration $(0,0025$ p. 100$)$ elle prévient l'apparition de saveur oxydée dans les laits et les crèmes. Ajoutée à raison de 0,03 p. 100 à l'huile de beurre elle en retarde l'oxydation, si l'on en juge par l'évolution de l'indice de peroxyde (RICHARDSON, EL RaFeY et LoNG, 1947).

\section{Antioxygènes provenant des céréales}

\section{Farine d'avoine}

WAITE (1941) l'utilise à raison de 0,25 p. 100 dans une poudre de lait, qu'il peut ainsi conserver 4 mois à température ordinaire; une concentration de 0,5 p. 100 permet une conservation pendant une période deux fois plus longue mais la poudre présente alors un goût notable.

Hollender et Tracy (1942), JACK et Henderson (1942) obtiennent des résultats comparables avec des préparations commerciales (Avenex no 7, Avenol).

Davies (1940), Combs, Coulter et Whitman (1941) utilisent pour conditionner le beurre, des papiers dont une face est enduite de farine d'avoine et constatent une légère amélioration des qualités de conservation.

\section{Huile de germe de blé.}

Tracy, Hoskisson et Trimble (1944) améliorent la résistance du lait sec à l'autoxydation en y ajoutant de 0,05 à 0,2 p. 100 (calculé sur la matière grasse) d'huile de germe de blé. Ils notent l'action synergique de l'acide citrique et de la lécithine. Tls soulignent 
néanmoins, que l'amélioration obtenue est moindre que celle apportée par le conditionnement en boîte sous atmosphère inerte.

Chapman et Mac Farlane (1946) utilisent également l'huile de germe de blé comme antioxygène dans les laits secs, tandis que Godel (1949) utilise les huiles de germe de blé et de maïs, aussi bien dans les laits secs que dans les beurres.

\section{Tocophérol}

Considérés comme antioxygènes naturels et présents dans de nombreuses graisses, les tocophérols ne semblent pas avoir joué, dans les produits laitiers, le rôle que l'on attendait d'eux.

Nielsen et al. (1953) ajoutent des tocophérols à la ration de vaches laitières, sans obtenir d'amélioration des propriétés de conservation du beurre.

Mattill (1945), Richardson, El Rafey et Long (1947), REINART (1949), SWARTLING (1949) constatent que non seulement le rôle anti-oxydant des tocophérols n'est pas à retenir, mais qu'ils semblent parfois jouer le rôle de pro-oxydant. Toutefois, en combinaison avec des phospholipides (lécithine de Soja), Richardson, EL RAFEY et LONG (1947) leur trouvent une action retardatrice vis-à-vis de l'oxydation de l'huile de beurre.

\section{Antioxygènes préparés à partir du lait}

Nous ne ferons que mentionner la libération à partir des protéines, au cours du chauffage des laits, des dérivés sulfhydryles qui agissent comme antioxygène et nous parlerons plus en détail du procédé proposé par SıöJTRöm et LARsson (1949). Ces auteurs préparent un agent réducteur par traitements alcalins répétés du lactosérum, La solution ainsi obtenue est mise en poudre et incorporée au lait, au lait sec et au beurre.

THOME et OLsSON (1951) utilisent cet antioxygène connu en Suède sous le sigle de " $\mathrm{A} \mathrm{B} \mathrm{V} \mathrm{".} \mathrm{Ils} \mathrm{l'ajoutent} \mathrm{soit} \mathrm{à} \mathrm{la} \mathrm{crème,} \mathrm{soit}$ au beurre. Les beurres sont dégustés après un stockage à $13^{\circ} \mathrm{C}$ de 14 jours à 1 mois et après un stockage à $-20^{\circ} \mathrm{C}$ pendant 3 mois. Si la préparation semble avoir un pouvoir antioxydant marqué, il ne l'est pas suffisamment dans certains cas, pour supprimer totalement les risques de saveur graisseuse.

REINART et BRown (1953) reprenant les travaux de Coombs (1949) utilisent également une poudre de sérum comme antioxygène.

Mac Dowell (1955) prépare un antioxygène, selon la technique suédoise, mais à partir de lait écrémé. Il l'ajoute à raison de 1 p. 100 
dans le beurre. Il en résulte une coloration et une saveur de caramel ; celle-ci disparaît peu à peu. Au bout de huit mois de conservation, le "goût de frigo " (storage flavour) n'est pas moins intense dans les beurres contenant le produit actif que dans les beurres témoins. Une différence apparaît toutefois si, après ce délai, on garde les beurres à la température de $13^{\circ} \mathrm{C}$, pendant 15 jours.

\section{2 - Antioxygènes de synthèse.}

\section{Gallates d'alkyle}

Essayant d'empêcher l'apparition de saveur oxydée dans le lait, AULe et Storgårds (1953), ajoutent, au cours d'une expérience de sept semaines, 10 grammes de gallate de propyle à la ration des vaches laitières, sans réussir à améliorer les qualités organoleptiques du lait.

Chilson, Martin et Whitnah (1950) ajoutent $20 \mathrm{mg} / 1 \mathrm{de}$ gallate de propyle à un lait, qui conservé pendant 14 jours à la température de $+1^{\circ} \mathrm{C}$, en présence de $0,5 \mathrm{ppm}$ de cuivre ne prend aucune saveur oxydée.

\section{TABLEAU II}

INFLUENCE DE DIFFÉRENTS ESTERS DE L'ACIDE GALLIQUE AJOUTÉS A LA DOSE DE 0,001 P. 100 SUR L'ÉVOLUTION DE L'INDIGE DE PEROXYDE (I) DE LA MATIËRE GRASSE D'UN LAIT SEG ENTIER (D'APRÉS TOLLENAAR, 1949)

\begin{tabular}{|c|c|c|c|c|}
\hline & \multicolumn{4}{|c|}{ Jours de stockage à $30^{\circ} \mathrm{C}$} \\
\hline & 10 & 40 & 70 & 125 \\
\hline Témoin $\ldots \ldots \ldots \ldots \ldots \ldots \ldots$ & 3,6 & 5,9 & 83 & 291 \\
\hline Acide gallique ............ & 9,6 & 218 & 353 & 362 \\
\hline Gallate de méthyle ......... & 2,7 & 6,7 & 102 & 294 \\
\hline Gallate d'octyle $\ldots \ldots \ldots \ldots$ & 3,0 & 2,9 & 3,5 & 13,6 \\
\hline Gallate de tétradécyle ....... & 2,3 & 2,4 & 2,9 & 4,2 \\
\hline
\end{tabular}

(1) Indice de peroxyde exprimé en méq. d'oxygène actif par kg de matière grasse.

En 1945, Findlay, Smith et Lea, constatent que le gallate d'éthyle à raison de 0,06 à 0,08 p. 100, protège la poudre de lait contre la saveur oxydée et n'apporte aucun goût. Wніте, Sмгтн et LEA (1947) confirment ce résultat précisant qu'un préchauffage du lait à $88^{\circ} \mathrm{C}$ et l'addition de 0,08 p. 100 de gallate, permettent de prolonger trois fois la durée du stockage à $47^{\circ} \mathrm{C}$ et huit fois celle 
d'un stockage à $15^{\circ} \mathrm{C}$. Par contre, ils considèrent les esters propylique, butyrique et amylique comme inutilisables en raison du goût amer qu'ils donnent à la poudre.

TollenaAR (1949) compare les effets de plusieurs esters, ajoutés à raison de 0,001 p. 100 dans le lait avant dessiccation par l'évolution de l'indice de peroxyde de la partie grasse, en fonction de la durée de conservation (tableau II).

Les esters supérieurs de l'acide gallique semblent donc donner pleinement satisfaction. D'ailleurs TollenaAR confirme, en 1952, que 0,01 p. 100 de gallate de tétradécyle permet de conserver sans dommage une poudre de lait, einq fois plus longtemps qu'une poudre témoin.

Dès 1944, LEA signale que les esters de l'acide gallique apportent une protection efficace contre l'autoxydation du beurre sans causer la décoloration ou apporter de saveur étrangère : ainsi 0,005 p. 100 de gallate d'éthyle ajoutés à un beurre conservé à la température de $37^{\circ} \mathrm{C}$, retardent considérablement l'apparition d'une saveur suiffeuse.

En Australie, Pont (1945) constate qu'un beurre salé à 3 p. 100 et contenant 0,02 p. 100 de gallate d'éthyle, conservé en boîte, résiste beaucoup mieux à l'autoxydation qu'un témoin.

SwartLing (1949) souligne l'efficacité du gallate d'éthyle $(0,0025$ p. 100) pour retarder l'apparition d'une saveur graisseuse, pendant une courte période de stockage à la température de $13^{\circ} \mathrm{C}$; mais à la température de $-18^{\circ} \mathrm{C} 1^{\prime}$ 'antioxygène est sans effet. Utilisés dans du beurre de sérum, les gallates d'octyle et de dodécyle inhibent l'oxydation mais provoquent une coloration pourpre (New-Zealand Dairy Res. Inst., 1957-58).

L'action favorable du gallate de dodécyle n'est pas confirmée par Van Haeften et Pette (1953). Thomé (1953) signale que les gallates, même associés à des citrates, n'agissent plus à la fin d'une longue période de stockage. TollenaAR $(1951,1952)$ souligne que les esters de l'acide gallique amoindrissent les effets de l'oxydation, si l'on en juge par des tests chimiques, mais ne suppriment pas les défauts de saveur des beurres stockés à $-8^{\circ} \mathrm{C}$ ou entre -15 et $-24^{\circ} \mathrm{C}$.

Mac Dowell (1955) aboutit à la même conclusion.

\section{Acide ascorbique}

L'acide ascorbique (Vitamine C) ou ses dérivés (palmitate d'ascorbyle) ont été utilisés avec un certain succès comme antioxygène, soit pour préserver le lait de consommation (CHILson, MARTiN, Parrish, 1949), soit dans les laits destinés à être desséchés : Coul- 
TER (1944) signale que 0,1 p. 100 d'acide ascorbique (calculé sur la poudre) retarde l'oxydation; Findlay, Smith et LeA (1945) constatent que la durée du stockage à la température de $37^{\circ} \mathrm{C}$ peut être allongée sans qu'il y ait modification du goût ou de la couleur du produit, grâce à l'addition de 0,003 à 0,03 p. 100 d'acide ascorbique. WRIGHT et GREENBANK (1949) confirment ces résultats.

Smith et al. (1952) ajoutent de 0,005 à 0,01 p. 100 d'acide ascorbique à des crèmes qui sont conservées pendant 9 mois à la température de $-22^{\circ} \mathrm{C}$, sans que des défauts de saveur apparaissent.

Expérimentant sur le beurre REINART et BRown (1953) ajoutent de 0,015 à 0,02 p. 100 d'acide ascorbique : le beurre est conservé pendant huit mois, avant qu'une altération de la saveur apparaisse. MAC Dowell (1955) signale que, si l'anti-oxygène est efficace à raison de 0,1 à 0,2 p. 100 , un goût de noix de coco très prononcé rend la commercialisation du beurre impossible. De même BudsLAWSKI (1958) qui réussit à conserver du beurre en parfait état de fraîcheur pendant 12 mois note qu'il apparaît, après une longue période de conservation, une saveur " maltée ", accompagnée d'un brunissement.

\section{TABLEAU III}

ACTION DU TMTD SUR LA GONSERVATION D'UN BEURRE MAINTENU A - 12' G (EXAMEN ORGANOLEPTIQUE ET ANALYSE GHIMIQUE)

(D'APRÉS TOLLENAAR, 1951)

\begin{tabular}{|c|c|c|c|c|c|c|}
\hline & \multicolumn{2}{|c|}{ Témoin } & \multicolumn{2}{|c|}{ T M T D 0,005 p. 100} & \multicolumn{2}{|c|}{ T M T D 0,015 p. 100} \\
\hline & Note & I. P. (1) & Note & I. P. & Note & I. P. \\
\hline $0 \ldots \ldots \ldots$ & 6,5 & 0,0 & 5 & 0,2 & - & 0,8 \\
\hline 4 mois ... & 4 & 2,2 & 5,5 & 0,2 & 5 & 1,4 \\
\hline 5 mois ... & 3,5 & 2,4 & 5,5 & 0,5 & 5,5 & 0,9 \\
\hline 6 mois ... & 3,5 & 3,0 & 5,5 & 0,6 & 5,5 & 1,2 \\
\hline
\end{tabular}

(1) Indice de peroxyde exprimé en méq. d'oxygène actif par kg de matière grasse.

TollenaAR (1952) a également constaté l'apparition d'un goût désagréable et très prononcé. SмrтH et al. (1952) ne retiennent pas l'acide ascorbique comme antioxygène puisque des beurres, contenant 0,02 p. 100 d'acide, présentaient une saveur oxydée plus intense que celle du témoin. 


\section{Disulfure de tetraalkylthiurame}

En 1951, Tollenaar propose l'utilisation de disulfure de tétraéthylthiurame (TETD) et du disulfure de tétraméthyl thiurame (TMTD) qui, à des concentrations de 0,005 à 0,015 p. 100 , montrent une action favorable sur la conservation des beurres préparés à partir de crèmes acides et conservés à la température de $-12^{\circ} \mathrm{C}$. L'examen organoleptique corrobore ce résultat (tableau III).

Van Haeften et Pette (1953) confirment l'efficacité du TETD.

Cependant, ces composés apportent une légère saveur étrangère (TollenaAR, 1951 ; Mac Dowell, 1955). Le TETD serait efficace lorsqu'on l'utilise incorporé aux crèmes congelées (Mac DoweLL, 1955).

\section{Diéthyldithiocarbamate de sodium (Na DEDC)}

Le Na DEDC inhiberait la formation de saveur oxydée dans les beurres mais donnerait une saveur étrangère (Mac DowELL, 1955).

\section{Hydroquinone}

L'hydroquinone se révèle être un antioxygène efficace incorporée aux laits en poudre (WAITE, 1941) mais à la concentration de $0,5 \mathrm{p}$. 100 elle donne un goût métallique accusé. MưLder, KLeikamp et KING (1947) estiment qu'à raison de $20 \mathrm{mg} / 1$ elle prévient l'apparition de défauts de goût dans les crèmes.

Notons cependant que les produits d'oxydation de l'hydroquinone sont toxiques.

\section{2 et 3 butyl 4 hydroxyanisol (BHA) et 2-6 dibutylparacrésol ou butylhydroxytoluène ( $B H T$ )}

Ces deux composés sont les antioxygènes aujourd'hui les plus utilisés aux Etats-Unis; pourtant peu d'études ont été faites sur leur utilisation dans les produits laitiers.

Gelpi, Rusoff et Skole (1952) signalent que le BHA utilisé seul ou en combinaison avec l'acide citrique et le gallate de propyle empêche l'apparition de saveur oxydée pendant au moins six mois dans lès crèmes congelées. TollenaAR et Vos (1956) incorporent du $\mathrm{BHT}$, à des laits secs et à des beurres, obtenant de bons résultats. Des travaux anonymes, cités par le New Zealand Dairy Res. Inst. (1957-1958), signalent qu'à la concentration de 0,016 p. 100, le $\mathrm{B} \mathrm{H} \mathrm{A}$ ne retarde pas l'oxydation de beurre de sérum conservé à la température de $-10^{\circ} \mathrm{C}$, tandis qu'à la même concentration le BHT est efficace. 
DE LUCA (1957) ajoute du BHT à la ration des vaches laitières. Le composé est mis en évidence et dosé dans le lait mais ne protège pas de l'oxydation.

Signalons pour terminer que DUGAN et al. (1950) proposent d'utiliser le $\mathrm{B}$ H A pour imprégner les papiers destinés à emballer les matières grasses.

\section{Produits divers}

D'autres antioxygènes ont également été proposés. Citons :

- le trihydroxybutyrophérone (STUCKey et GEarhaRT, 1957), l'acide citraconique (Mac Dowell, 1955), le caffeate d'éthyle (GELPI et al., 1952).

\section{IV. - DISGUSSION}

Quelques résultats semblent avoir été obtenus par l'adjonction d'antioxygènes aux laits secs. En ce qui concerne les beurres, l'addition d'antioxygènes ne semble pas avoir comblé les espérances de ceux qui pensaient pouvoir traiter le beurre comme un quelconque corps gras. Certains antioxygènes, réputés efficaces, n'ont produit aucun effet bénéfique; parfois même ils communiquent un goût désagréable à la matière grasse. D'autres antioxygènes semblent améliorer les qualités de conservation du beurre. Pour juger de leur efficacité, deux méthodes ont été utilisées : l'une consiste à rechercher si la saveur désagréable que l'on cherchait à faire disparaître s'est au moins atténuée ; l'autre consiste à comparer le degré d'oxydation, estimé par voie chimique, avec celui d'un témoin. Cette seconde méthode, si elle est utile, n'est pas suffisante pour améliorer la saveur d'un produit alimentaire, d'autant plus que nous travaillons dans un pays où le consommateur est exigeant sur ce point.

En supposant que l'on trouve un antioxygène, inodore, sans saveur, ne produisant aucune altération de la couleur du produit et surtout totalement dénué de toxicité, on peut tout de même se demander si, à une époque où le public semble manifester un engouement pour les huiles "vierges ", ce n'est pas faire fausse route que de vouloir ajouter un antioxygène au beurre. En effet l'une des chances de ce produit, face à l'offensive des corps gras artificiels, est qu'il reste aux yeux du consommateur le symbole du produit naturel. L'addition d'une substance étrangère, si efficace soit-elle, ne va-t-elle pas le rabaisser au niveau des autres graisses alimentaires?

Ne conviendrait-il pas aussi de rappeler que "la neutralisation de l'acidité est encore l'arme la plus efficace que l'on ait découverte, 
contre les défauts d'oxydation des beurres de crèmes acides " (Тноме, 1953).

Cette plus grande sensibilité des beurres de crèmes acides à l'autoxydation, pourrait amener à concevoir une orientation nouvelle de la technique beurrière française ; sans pour autant renoncer à la fabrication des beurres aromatiques, les industriels pourraient éviter de les stocker, et s'efforcer de ne conserver que des beurres naturellement moins fragiles.

\section{RÉFÉRENCES BIBLIOGRAPHIQUES}

O. AULe et T. Storgards, The effect of feeding propyl gallate to dairy cows on the development of oxidized flavour in milk. XIIIth Intern. Dairy Cong. The Hague, 1953, 2, 112.

J. BUdslawski. Etude sur l'utilisation de l'acide aseorbique et de ses dérivés comme antioxygènes du beurre. Cahiers d'études. Ecole Supérieure d'Agriculture. Olsztyn (Pologne), 1958, 8, n $\mathrm{n}^{0} 60$.

R. A. Chapman et W. D. McFarlane. The effet of wheat germ oil antioxidants and natural reducing substances on the stability of whole milk powder. Can. Journ. Res., 1946, 24F, 47, cité par D. S. A., 1946-47, $8(2), 136$.

W. H. Chilson, W. H. Martin et C. H. Whitnah. Use of propyl gallate to defer development of oxidized flavor in market milk. J. Dairy Sci., $1950,33,925$.

W, H. Chilson, W. H. Martin et D. B. Parrish. The relationship of ascorbic acid to the development of oxidized flavor in market milk. J. Dairy Sci., 1949, 32, 306.

W. B. Combs, S. T. Coulter et D. W. Whitman. Avenized versus standard parchment for wrapping print butter. J. Dairy Sci., 1941, 24, 117.

G. W. Cоомвs. The Australian Milk and Dairy Products Journal, 1949, 16, 12, 10, cité par Reinart A. et R. W. Brown, 1953.

S. T. Codlter. Deshydrated milk and milk products. Use of antioxidants. J. Dairy S'ai, 1944, 27, 607 (Annual meeting).

S. T. Coulter, R. Jenness et W. F. Geddes. Physical and chemical aspects of the production, storage and utility of dry milk products. Advances in Food Research, 1951, 3, 45.

E. A. DAY. Autoxidation of milk lipids. J. Dairy Sci., 1960, 43, 1360.

W. L. Davies. Antioxygenic offect of cereal flour paste as coating in contact wrappers for fatty foods. J. Indian Chem. Soc. (Ind. Ed.), 1940, 3, 174-182, cité par D. S. A., 1945-46, 7 (2), 146.

L. R. Dugan Jr., H. R. Kraybill, L. Ireland et F. C. Vibrans. Butylated hydroxyanisole as an antioxidant for fats and foods made with fat. Food Technology, 1950, 4 (11), 457.

J. D. Findiax, J.A. B. Smith, G. H. Lea. Experiments on the use of antioxidants in spray dried whole milk powder. J. Dairy Res., 1945, 14,165 . 
A. J. Gelpi, L. L. Rugoff et R. D. Skole. Effect of antioxidants in the control of oxidized flavor development in stored frozen cream. I. Use of Ethyl caffeate, Sustane, Tenox II and Tenox B H A. J. Dairy Sci., $1952,35,93$.

A. Godel. Vitaminy Kak sredstwo borby s progorkaniem zira. Mol. Prom., 1949, 10 (7), 28, cité par D.S.A., 1950-51, 12 (1), 80.

C. Goldmbic. Oil and Soap, 1946, 24, 184, cité par Spetsig, 1960.

H. A. Hollender et P.H. Tracy. The relation of the use of certain antioxidants and methods of processing to the keeping quality of powdered whole milk. J. Dairy Sci., 1942, 25, 249.

E. L. JACK et J. L. HENDERson. Preventing off-flavors in dried whole milk. Food Inds, 1942, 14 (3), 50, cité par S. I. Coulter, R. Jenness et W. F. GedDes, 1951.

V. N. Krukowsky, A. T. Dionisios, F. Whiting et E. S. Guthrik. The effect of nordihydroguaiaretic acid, salt and temperature of storage on the stability of fat, and fat soluble vitamins in cream and butter. J. Dairy Sci., 1949, 32, 679.

C. H. Lea. Experiments on the use of antioxidants in dry edible fats. J. Soc. Chem. Ind., 1944, 63 (4), 107, cité par D. S. A., 1944-45, 6 (2), 102.

C. H. LEA. Teehnological aspects of antioxidants. J. of Science of Food and Agrioulture, 1949, 9, 627.

A. P. de Luca, R. Teichman, J. E. Rousgeau, M. E. Morgan, H. D. Eaton, P. Macleod, M. W. Dicks et R. E. Johnson. Relative effectiveness of various antioxidants fed to lactating dairy cows on incidence of copper induced oxidized milk flavor and on apparent carotene and tocopherol utilization. J. Dairy S'ci., 1957, 40, 877.

W. O. LundberG, H. O. Halvorson et G. O. Burr. The antioxidant properties of N D G A. Oil and Soap, 1944, 21 (2), 33, cité par J. Dairy Sci., 1944, 27, A94.

A. K. R. MACDowell. The effect of salt and of antioxidants on the keeping quality of butter. J. Dairy Res., 1955, 22, 349 .

H. A. Matrill. Antioxidants and synergists. Oil and Soap, 1945, 22, 1, cité par B. J. Morris. (The Chemistry and Technology of Food and Food Products. Interscience Publishers. Inc. New York), 1951, III, 1964.

C. Moureu et C. Dufraisse. Bull. Soc. Chim., 1922, 31 (4), 1152, cités par C. Dufraisse dans Grignard (Traité de Chimie Organique, Masson et Cie, éditeurs, Paris), 1936, II, 1147.

H. Mulder, J. H. B. Kleikamp et N. King. The acidity of cream affecting the flavour of eream and butter. Neth. Milk Dairy J., 1947, 1, 225.

New Zealand Dairy Research Institute. Effect of antioxidants on keoping quality of butter. Annual Report, 1949-50.

New Zealand Dairy Researoh Institute. Effect of antioxidants on quality of whey butter. Annual Report, 1957-58.

J. Nielden, A. N. Fisker, A. H. Pedergen, I. Prance, E. Sondergaard et H. DAM. The influence of feeding tocopherol to dairy cows on the yield of milk and milk fat and on the tocopherol content and keeping quality of the butter. J. Dairy Res., 1953, 20, 333 . 
H. S. Ozcotт et H. A. Matrint, $J$. Am. Chem. Soc, 1936, 58, 2204, cités par B. J. Monris (The Chemistry and Technology of Food and Food Products, Interscience publishers, Inc., New York), 1951, III, 1964.

E. G. Pont. The keeping quality of tinned butter. J. Conn. Sci. Industr. Res. Aust., 1945, 18 (1), 53, cité par D. S. A., 1945-46, 7 (2), 87.

A. Reinart. On the antioxidant properties of vitamin E. ( $\alpha$-tocopherol) synthetic $\alpha$-tocopherol and di- $\alpha$-tocopherol acetate in butterfat. XIIIth Intern. Dairy Cong., Stockholm, 1949, 2, 405.

A. Reinart et R. W. Brown. Production of a dairy spread of improved stability. XIIIth Intern. Dairy Cong. The Hague, 1953, 2, 522.

G. A. Richardson, M. S. El Rafey et M. L. Long. Flavones and flavones derivatives as antioxidants. $J$. Dairy $S_{c} i ., 1947,30,397$.

G. SJöströn et A. LARsson. The production of antioxidative substance from milk. XIIth Intern. Dairy Cong. Stockholm, 1949, 2, 368.

A. C. Smith, M. Loewenstein, R. E. Anderson et H. C. Olson. The role of ascorbic acid and tocopherol in the development of oxidized flavor in eream and butter. J. Dairy Sci., 1952, 35, 485 (M 24).

O. Spetsig. Antioxidant synergism by hydrogen transfer and addition reactions. Arkiv för Kemi, 1960, 15, 5 .

Ben N. Stuckey et W. M. Gearhart. Trihydroxybutyrophenone. A food grade antioxidant. Food Technology, 1957, 11, 676.

J. W. Stull, E. O. Herreid et P. H. Tracy. A study of the use of the antioxidant $\mathrm{NDG} A$ acid in dairy products. I. Its antioxigenic properties in milk. J. Dairy Sci., 1948a, 31, 449.

J. W. Stull, E.S. Herreid et P. H. Tracy. A study of the use of the antioxidant N D G A acid in dairy products. II. Its antioxygenic properties in unsweetened frozen cream. $J$. Dairy Sci., 1948b, 31, 1024.

J. W. Stuli, E. S. Herreid et P. H. tracx. A study of the use of the antioxidant N D G A acid in dairy products. IV. Its antioxygenic properties in spray dried whole milk and ice cream mix, with, and without, added synergists. J. Dairy Sci., 1951, 34, 80.

P. Swarting. The antioxidative effect of certain aromatic hydroxy compounds in butter with special reference to ethyl gallate. XIIth Intern. Dairy Cong., Stockholm, 1949, 21, 375.

N. P. TARAsiuk et J. L. Henderson. A water soluble antioxidant for milk and milk products. J. Dairy Sci., 1946, 29, 501.

K. E. Тноме. Changes in quality of butter during storage. XIIIth Intern. Dairy Cong., The Hague, 1953, 2, 499.

K. E. Тноме et T. Olsson. Buttermaking experiments using an antioxidant prepared from whey. Report $n^{0} 32$, State Dairy Res. Station. Alnarp (Suède), 1951.

F. D. Tollenaar. Occurence and prevention of oxidative deterioration in dairy products. XIIth Intern. Dairy Cong., Stockholm, 1949, 2, 357 et 7,73 .

F. D. Tollenaar. The inhibition of oxidative defects in cold stored butter by some tetra alkylthiuram disulfides. Netherl. Mitk Dairy J., 1951, 5,46 . 
F. D. Tollenan. Possibilities and limitations of the use of antioxidants in edible oils and fats. IXe Cong. Intern. Ind. Agric., Rome, 1952, T. P. 9.

F. D. Tollenaar et H. J. Vos. Use of butylated hydroxytoluene as an antioxidant in foods. Fette, Seifen Anstrichmitt, 1956, 58 (2), 112, cité par D. S. A., 1959, 21 (6), 272.

P. H. Tracy, W. A. Hoskisson et J. M. Trimble. Wheat germ oil as an antioxidant in dairy products. J. Dairy Sci., 1944, 27, 311.

G. M. Trout et B. R. Weinstein. The solar activated flavor of homogenized milk. XIII th Intern. Dairy Cong. The Hague, 1953, 3, 1061.

G. A. Ulex et E. .P. Kröger. Uber die Wirksamkeit der Nordihydroguajaretsäure als Antioxidant für Fette. Dtsch. Lebensmitt. Rdsch., 1950, 46 (11), 256, eité par D. S. A., 1951, 13 (4), 532.

F. E. Van Haeften et J. W. Pette. Cold storage experiments on butter. XIIIth Intern. Dairy Cong. The Hague, 1953, 2, 541.

R. WaIte. The keeping quality of milk powders. J. Dairy Res., 1941, 12, 178.

J. C. D. White, J. A. B. Smith et C. H. Lea. The effect of a high preheating temperature with and without ethyl gallate on the storage life of whole milk powder. J. Dairy Res., 1947, 15, 127.

P. A. Wright et G. R. Greenbank. The effect of the ascorbic acid content of fluid milk upon the keeping quality of its dried product. J. Dairy Sci., 1949, 32, 644.

\title{
CONTRIBUTION A L'ÉTUDE DE L'ACTION DE LA CHALEUR SUR LES CASÉINES (Fin)
}

\author{
par
}

Mme M. SWIRSKI, R. ALLOUF et H. CHEFTEL

Laboratoire de Recherches des Etablissements J.-J. Carnaud et Forges de Basse-Indre

\section{II. - Influence du chauffage sur l'hydrolyse enzymatique}

\section{A. - Hydrolyse trypsique}

\section{Mise en liberté d'azote aminé.}

Les dosages ont été effectués après $3,24,48,72$ et 100 heures d'hydrolyse. La figure 4 résume nos résultats : en l'absence de chauffage préalable, l' $\alpha$-caséine est hydrolysée bien plus rapidement que la caséine totale; le chauffage ralentit considérablement l'hydrolyse tant de l'une que de l'autre ; la $\beta$-caséine, au contraire, est relativement inerte vis-à-vis de la trypsine, ainsi que CHRIstensen [6] et 